\title{
Rotational and Vibrational Nonequilibrium in a Low Diffusion Particle Method for Continuum Flow Simulation
}

\author{
Jonathan M. Burt ${ }^{1}$ and Iain D. Boyd ${ }^{2}$ \\ Department of Aerospace Engineering \\ University of Michigan, Ann Arbor, MI 48109
}

\begin{abstract}
A low diffusion particle method for simulating compressible low Knudsen number gas flows is modified for application to flows involving nonequilibrium distributions in rotational and vibrational energy modes. This method is closely based on the direct simulation Monte Carlo (DSMC) method, and has been developed for use in a strongly coupled hybrid scheme with DSMC. In simulations employing this hybrid scheme, the proposed modifications allow greater consistency with DSMC and reduced information loss along continuum breakdown boundaries when significant internal energy nonequilibrium effects exist within continuum flow regions. Two different approaches for rotational and vibrational nonequilibrium are proposed; the first provides greater efficiency and reduced sensitivity to time step size, while the second utilizes standard DSMC energy exchange procedures and should be easier to implement in an existing DSMC code. Both approaches are evaluated through comparison with DSMC for a set of homogeneous relaxation problems, and capabilities of the hybrid scheme are demonstrated in simulations of a hypersonic flow over a cylinder.
\end{abstract}

\section{Introduction}

A wide range of gas flow problems of engineering interest involve regions of continuum flow as well as rarefied regions with strong translational nonequilibrium. These flow problems include gas flows within and around micro-electro-mechanical systems (MEMS), supersonic flows for which the internal structure of shocks is important, flows around reentry vehicles and other hypersonic atmospheric flows, gas venting into a near-vacuum, and rocket exhaust flows at high altitudes. In any of these types of flows, characteristic lines or diffusive quantities may travel in both directions between rarefied and continuum regions, so that two-way coupled information transfer is required during flowfield simulation. Rarefied regions are typically simulated using the direct simulation Monte Carlo (DSMC) method, ${ }^{1}$ the dominant method for simulation of high Knudsen number (Kn) gas flows.

In a DSMC simulation, a large number of particles - each representing a large collection of atoms or molecules are tracked through a computational grid. Particle move and collide operations during each simulation time step are consistent with advection and collision terms in the Boltzmann equation (the governing equation for dilute gas flows at any $\mathrm{Kn}$ ) and macroscopic flow properties are evaluated as cell-based averages of particle quantities. Relative to other simulation techniques for high Kn flows, DSMC tends to provide an excellent balance of computational efficiency, low memory and accuracy. While the underlying assumptions in DSMC are valid in both high and low Kn regimes, the DSMC method can be prohibitively expensive in low Kn regions due to severe restrictions on the cell size and time step interval. More efficient computational fluid dynamics (CFD) methods, typically based on a finite volume solution to the Navier-Stokes (NS) equations, are generally used instead for simulation of low Kn regions where the NS equations are valid.

\section{A. Hybrid Schemes for Rarefied and Continuum Flow Simulation}

When two-way coupling between rarefied and continuum flow calculations is required, a hybrid CFD-DSMC scheme is typically employed. This type of scheme involves integration of separate DSMC and CFD algorithms into a single code, with domain decomposition into DSMC and CFD regions through evaluations of a continuum breakdown parameter and coupled information exchange along breakdown boundary surfaces. In weakly coupled hybrid approaches, suitable for steady state flow simulation, calculations are alternately performed in DSMC and

\footnotetext{
${ }^{1}$ Post-doctoral research fellow, AIAA member.

${ }^{2}$ Professor, AIAA associate fellow.
} 
CFD regions, and each calculation is allowed to relax somewhat toward steady state before boundary conditions associated with the other calculation are updated..$^{2-5}$ Strongly coupled hybrid approaches involve more frequent updates to these boundary conditions, and can allow for unsteady flow simulation while avoiding potential errors due to fluctuations in quantities transported across continuum breakdown boundaries. ${ }^{6-9}$

In recent years, a variety of hybrid CFD-DSMC schemes have been developed and applied to a range of test cases. Most of these test cases involve relatively simple flow configurations, and have been used primarily for assessment of simulation accuracy and efficiency. While CFD-DSMC hybrid schemes should also allow for accurate simulation of more complex flows for which other simulation techniques are either inaccurate or prohibitively expensive, to date these schemes have been utilized very little as engineering design and analysis tools. One reason for this may be the inherent complexity of such schemes; integration of DSMC and CFD codes tends to require extensive development time - even if the developer has access to two pre-existing codes - and coupling procedures are generally complex and difficult to implement in parallel.

In an effort to develop simpler alternative DSMC-based techniques for coupled simulation of flows involving both high and low Kn regions, a number of authors have proposed modifications to DSMC for application to low Kn regimes. ${ }^{10-15}$ The proposed DSMC-based particle methods can be categorized as either collision limiters or as direct resampling techniques. The first type of method involves a specified upper limit on the number of collisions per particle or per cell during each time step, ${ }^{10,11}$ while in the second type of method either all or some fraction of particle velocities are resampled during each time step from desired distribution functions. ${ }^{12-15}$ While these DSMCbased low Kn methods vary greatly in complexity, they are all considerably simpler and easier to implement in an existing DSMC code than proposed hybrid CFD-DSMC schemes. In contrast to a hybrid CFD-DSMC algorithm, a hybrid technique utilizing a DSMC-based particle method in continuum regions and standard DSMC in rarefied regions allows for strongly coupled information exchange at continuum breakdown boundaries through simple particle advection, and there is no requirement for integration of two different codes. In addition to straightforward procedures for strong two-way coupling ${ }^{13,14}$ and a relatively small source code, other advantages include greatly simplified parallelization and the potential for a unified approach to internal energy nonequilibrium through a DSMC-like treatment of rotational or vibrational energy in continuum particle methods.

Despite such advantages of an "all-particle" hybrid scheme, inherent problems with DSMC-based continuum flow simulation methods generally make this type of scheme impractical. In particular, random particle motion occurs over length scales comparable to the cell size, which leads to large artificial diffusion and dissipation effects when the cell size and time step are much larger than the mean free path and mean collision time respectively. ${ }^{10,16}$ This means that, for accurate simulation of continuum flows with boundary layers, slip lines or other regions involving strong transverse gradients, these DSMC-based methods have similar cell size and time step requirements as standard DSMC and provide only marginal efficiency benefits.

\section{B. The Low Diffusion Particle Method}

As an alternative basis for a hybrid scheme with DSMC, combining the accuracy of a hybrid CFD-DSMC scheme with the simplicity of an all-particle scheme, a new DSMC-based low diffusion (LD) particle method has recently been developed by the authors. ${ }^{17-20}$ This method is based on an approximation of an inviscid compressible flow as a collection of contiguous finite volume equilibrium reservoirs, represented by Lagrangian cells, which are bounded by massless specularly reflecting walls corresponding to each Lagrangian cell face. Kinetic theory arguments are used to determine the velocity of each face, and particle velocities are updated during each time step so that every particle remains fixed with respect to the assigned Lagrangian cell over the time step interval. ${ }^{17}$ Particle trajectories therefore tend to follow gas streamlines, as is physically appropriate for an inviscid flow on cell-based length scales much larger than the mean free path. A temperature and a separate "bulk" velocity - used to assign thermal energy and momentum respectively - are given to each particle, and both quantities are determined through kinetic theory expressions to enforce momentum and energy conservation.

In an LD particle method simulation, standard DSMC procedures (with some minor modifications) are used for particle movement, Eulerian cell assignment and time-averaged sampling in each cell, while the following routines are performed during each simulation time step in place of DSMC collision calculations:

1. The density, bulk velocity and temperature are evaluated in each fixed Eulerian cell as functions of the cellaveraged particle properties, and these values are stored in the cell data structure.

2. A velocity is calculated for all faces of each Lagrangian cell, using a series of expressions derived from kinetic theory. Lagrangian cells are coincident with Eulerian cells at the beginning of each time step, but then move and deform over the time step interval according to local gas properties. 
3. Bulk velocity and temperature values assigned to each cell are updated, based on the contribution of momentum and energy transfer across all faces of the corresponding Lagrangian cell.

4. All particles in each cell are assigned the cell-based bulk velocity and temperature.

5. Velocities used for particle movement are updated, in such a way that all particles maintain a constant relative position within a Lagrangian cell over the time step interval.

The reader is referred to Ref. 17 for an outline of numerical procedures and further information on the LD particle method.

Through a series of detailed comparisons, considerable improvements in accuracy and efficiency have been demonstrated for the LD method over existing DSMC-based methods. For given levels of accuracy and precision, the LD method was found to be from one to three orders of magnitude more efficient than existing particle methods. ${ }^{17}$ In subsequent work, a strongly coupled LD-DSMC hybrid algorithm has been developed and tested. ${ }^{18}$ The method has also been extended for simulation of viscous low Kn flows through a simple finite volume calculation procedure for the viscous terms in the NS equations. ${ }^{19}$ Additional modifications include the use of a model for temperature and velocity slip along wall boundaries, ${ }^{19}$ incorporation of mixing rules for transport coefficients in a gas mixture, and the addition of species diffusion velocities to cell face velocities for inclusion of mass diffusion effects. ${ }^{20}$

\section{Rotational and Vibrational Nonequilibrium}

In the current effort, modifications are proposed to the LD particle method for nonequilibrium characterization of rotational and vibrational energy. Previous versions of the LD method have assumed thermal equilibrium, with full accommodation of internal energy modes to the translational temperature and a fixed number of internal degrees of freedom. While these are generally accurate approximations for low temperature flows at very low Kn, both thermal nonequilibrium and vibrational excitation effects may become significant at higher temperatures and higher $\mathrm{Kn}$. In particular, very large vibrational collision numbers allow for potentially large differences between vibrational temperatures and the corresponding translational temperatures, while permitting highly nonequilibrium quantized vibrational energy distributions even in very low Kn regimes. At somewhat higher Kn rotational nonequilibrium may also important; both rotational temperature lag and nonequilibrium rotational energy distributions can be observed within shocks or regions of rapid expansion.

Vibrational and rotational nonequilibrium effects are typically included in CFD simulations of high enthalpy hypersonic flows through the use of separate vibrational, rotational and translational temperatures, although often a single translational/rotational temperature is employed to reduce simulation cost and complexity. ${ }^{21} \mathrm{An}$ alternative "ladder-climbing" technique for vibrational excitation permits characterization of nonequilibrium vibrational energy distributions by treating each quantum energy level as a separate species, but this is seldom used due to its additional complexity, computational expense and memory requirements. A proposed two-level model for vibrational excitation provides for a simplified and less costly representation of nonequilibrium energy distributions. However, this model considers only a limited range of energy distributions and involves simplifying assumptions which may not be valid for expanding flows. ${ }^{22}$

For either vibrational temperature or ladder-climbing quantum energy level based approaches, a loss of vibrational energy information is unavoidable along continuum breakdown boundaries in hybrid CFD-DSMC simulations due to inherent differences between DSMC and CFD methods. In a typical hybrid CFD-DSMC calculation employing vibrational energy excitation, newly generated DSMC particles at a continuum breakdown boundary are assigned vibrational energy values which are either sampled from an equilibrium Boltzmann distribution, or are set to some average energy level at the local CFD vibrational temperature. Subsequent CFD calculations then use vibrational temperature boundary conditions taken as the time-averaged local DSMC vibrational temperature. While to the authors' knowledge the ladder-climbing approach has never been implemented in a hybrid CFD-DSMC scheme, the extremely large scatter usually found in DSMC calculations of high vibrational energy level populations would likely result in a prohibitive level of noise in CFD vibrational energy boundary conditions along a continuum breakdown surface.

Similarly, in hybrid CFD-DSMC simulations involving diatomic or polyatomic gas species, an equilibrium rotational energy distribution is typically imposed along continuum breakdown boundaries between CFD and DSMC domains. For newly generated DSMC particles along these boundaries, rotational energy values are sampled from an equilibrium distribution at the local rotational or translational/rotational temperature in the CFD calculations. No information regarding the shape of the rotational energy distribution in DSMC cells along these boundaries is shared with the CFD module in the hybrid code, and some associated loss of accuracy is assumed. 
The hybrid LD-DSMC scheme described above, or any other hybrid scheme employing a DSMC-based particle method in continuum regions, allows for consideration of rotational and vibrational nonequilibrium in a manner that is both simpler and potentially more accurate than approaches used in hybrid CFD-DSMC simulations. In such allparticle hybrid schemes, particles which move between rarefied and continuum regions may carry their assigned rotational and vibrational energies across the boundary between the two regions. No information regarding the shape of internal energy distribution functions is lost in this process, and nonequilibrium energy distributions can be maintained in continuum regions through the same efficient Monte Carlo representation as in standard DSMC.

The main difficulty with inclusion of internal energy nonequilibrium in a DSMC-based continuum method, as outlined above, is in consideration of rotational and vibrational energy relaxation processes. Intermolecular collisions tend to force the internal energy distributions toward an equilibrium Boltzmann distribution, with redistribution of energy between internal and translational modes to enforce energy equipartition at equilibrium. Energy redistribution between different modes, and between different levels within each internal mode, must be included in calculation procedures. The assignment of a temperature value to each particle makes such energy redistribution procedures particularly simple in the LD method.

In the following sections, two approaches are proposed for rotational and vibrational nonequilibrium in the LD method, and a series of homogeneous relaxation test cases are used to demonstrate relative advantages in efficiency and accuracy for each approach. Next, results are presented for hybrid LD-DSMC simulations of a hypersonic flow over a cylinder with nonequilibrium rotational and vibrational excitation, and both capabilities and limitations of the hybrid scheme are demonstrated through comparison with DSMC simulation results. Finally, conclusions are presented, and areas of current and future work are discussed.

\section{Models for Internal Nonequilibrium in the Low Diffusion Method}

In this section, two different approaches are outlined for inclusion of rotational and vibrational nonequilibrium in the LD particle method. As described below, both approaches have relative advantages and weaknesses (in particular, the first tends to provide better efficiency while the second will in some situations have better accuracy) and neither is proposed as the optimal technique for all applications of the LD method. For both approaches, rotational and vibrational energy values $\varepsilon_{r}$ and $\varepsilon_{v}$ are assigned to each newly generated particle along inflow boundaries exactly as in standard DSMC, and these values are sampled from equilibrium distributions at the imposed inflow temperature. ${ }^{1}$ Also for both approaches, the temperature value $T$ assigned to each particle represents only the translational temperature, and the cell-averaged temperature as calculated in step (1) of the above LD method procedures ${ }^{17}$ is found (as for a monatomic gas) independently of any internal degrees of freedom. Furthermore, during particle cloning procedures related to information exchange between LD and DSMC calculations in a hybrid simulation, ${ }^{18}$ internal energy values $\varepsilon_{r}$ and $\varepsilon_{v}$ from parent particles are assigned to the newly generated clones.

\section{A. First Approach}

In developing the first approach for rotational and vibrational nonequilibrium in the LD particle method, we begin with Jeans' equation for homogeneous adiabatic relaxation of internal energy. ${ }^{24}$ Given an internal energy mode with an average energy value $e$ and $\zeta$ degrees of freedom, we can express the rate of relaxation toward the internal energy $e^{*}$ corresponding to the instantaneous translational thermal energy $e_{t}$ as

$$
\frac{d e}{d t}=\frac{e^{*}-e}{\tau}
$$

where $\tau$ is the characteristic internal energy relaxation time. If $\zeta$ is assumed constant (i.e. independent of $e$ ) then $e^{*}$ and $e_{t}$ may be related by $e^{*} / e_{t}=\zeta / 3$. Next, we denote initial values of the average internal and translational energies $e$ and $e_{t}$ as $e^{o}$ and $e_{t}^{o}$ respectively. From energy conservation arguments, it follows that $e+e_{t}=e^{o}+e_{t}^{o}$; hence

$$
e^{*}=\frac{1}{3} \zeta\left(e_{t}^{o}+e^{o}-e\right)
$$

Substitution of Eq. (2) into Eq. (1) then gives 


$$
\frac{d e}{d t}=\frac{C}{\tau}\left(\frac{\zeta\left(e_{t}^{o}+e^{o}\right)}{3 C}-e\right)
$$

where $C \equiv 1+\zeta / 3$. If the relaxation time $\tau$ is assumed constant, then Eq. (3) can be integrated over a finite time interval $\Delta t$ to express $e$ as a function of $e^{o}$ and $e_{t}^{o}$ :

$$
e(\Delta t)=\frac{\zeta}{3 C}\left[1-\exp \left(-C \frac{\Delta t}{\tau}\right)\right] e_{t}^{o}+\left[1-\frac{1}{C}\left(1-\exp \left(-C \frac{\Delta t}{\tau}\right)\right)\right] e^{o}
$$

We next define $\left(e^{*}\right)^{o}$ as the average internal energy at equilibrium with the initial translational energy $e_{t}^{o}$. Thus $\left(e^{*}\right)^{o} / e_{t}^{o}=\zeta / 3$ and substitution into Eq. (4) gives the final result

$$
e(\Delta t)=P \times\left(e^{*}\right)^{o}+(1-P) \times e^{o}
$$

where

$$
P \equiv \frac{1}{C}\left[1-\exp \left(-C \frac{\Delta t}{\tau}\right)\right]
$$

The parameter $P$ in Eq. (5) may be interpreted as the fractional contribution of $\left(e^{*}\right)^{o}$ to the internal energy $e$ after an elapsed time $\Delta t$. In other words, given the approximations of constant $\tau$ and $\zeta$, the average energy $e$ can be found at time $\Delta t$ by resampling a fraction $P$ of gas molecule internal energy values from an equilibrium distribution at the initial translational temperature.

Eq. (5) may be applied within LD particle method procedures to account for relaxation of rotational and vibrational energy distributions toward equilibrium. In the following, these two relaxation processes are considered independently. For each particle in a LD method simulation representing diatomic or polyatomic species, or each such particle within the LD domain in a hybrid LD-DSMC simulation, the rotational energy $\varepsilon_{r}$, vibrational energy $\varepsilon_{v}$ and temperature $T$ values in the particle data structure are updated during every time step just before step (1) in the LD method procedures outlined above.

Given a particle assigned to gas species $i$, we first determine the collision frequency $f$ and rotational collision number $Z_{r}$ for this particle. The collision frequency is calculated using the variable hard sphere (VHS) model of Bird $^{1}$ by summing over contributions from all simulated species $j$ :

$$
f=\sum_{j} \frac{1}{2}\left(d_{i}+d_{j}\right)^{2} n_{j}\left[2 \pi k_{B} T_{r e f}\left(\frac{m_{i}+m_{j}}{m_{i} m_{j}}\right)\right]^{1 / 2}\left(\frac{T}{T_{r e f}}\right)^{1-\omega}
$$

Here $d_{j}, m_{j}$ and $n_{j}$ are respectively the reference collision diameter, molecular mass and cell-based local number density for species $j$; $T_{\text {ref }}$ is the VHS reference temperature; $\omega$ is the VHS temperature exponent for viscosity; and $k_{B}$ is Boltzmann's constant. The rotational collision number is computed as a function of the particle temperature $T$, a reference temperature $T^{*}$ and a maximum collision number $Z_{\max }$ using the model of Parker. ${ }^{23}$

$$
Z_{r}=\frac{Z_{\max }}{1+\frac{1}{2} \pi^{3 / 2}\left(T^{*} / T\right)^{1 / 2}+\left(\pi+\frac{1}{4} \pi^{2}\right) T^{*} / T}
$$

Once $f$ and $Z_{r}$ are known for this particle, the probability of rotational-translational energy exchange $P_{r}$ is calculated as

$$
P_{r}=\frac{1}{C_{r}}\left[1-\exp \left(-C_{r} \frac{f \Delta t}{Z_{r}}\right)\right]
$$


where $C_{r}=5 / 3$ for a diatomic gas. Given a random number $R$ between 0 and 1, rotational-translational energy exchange will occur if $P_{r}>R$, in which case the particle rotational energy is resampled from an equilibrium distribution at the temperature $T$. For a diatomic gas, the new rotational energy is computed using the expression

$$
\varepsilon_{r}=-k_{B} T \ln (R)
$$

where $R$ denotes the output of another call to the random number generator.

Next, for consideration of vibrational-translational energy exchange, we first calculate the vibrational relaxation time $\tau_{v}$ as a function of the particle temperature $T$. An initial relaxation time $\tau_{M W}$ is found using the correlation of Millikan and White. ${ }^{25}$

$$
\tau_{M W}=\frac{\exp \left(A T^{-1 / 3}+B\right)}{P}
$$

where

$$
\begin{aligned}
& A=0.00116\left(\frac{1}{2} N_{A} m_{i}\right)^{1 / 2} \theta_{i}^{4 / 3} \\
& B=-0.015 A\left(\frac{1}{2} N_{A} m_{i}\right)^{1 / 4}-18.42
\end{aligned}
$$

Here $P$ is the cell-based local pressure in units of atm; $\theta_{i}$ is the characteristic vibrational excitation temperature in $\mathrm{K}$ for the particle species $i ; N_{A}$ is Avogadro's number in $\mathrm{kmol}^{-1}$; the species mass $m_{i}$ is in units of $\mathrm{kg}$; and the relaxation time $\tau_{M W}$ is given in s. A modified total relaxation time is then computed using the correction of Park ${ }^{26}$ for improved accuracy at high temperatures:

$$
\tau_{v}=\tau_{M W}+\frac{1}{\sigma_{v} n_{t o t}}\left(\frac{\pi m_{i}}{8 k_{B} T}\right)^{1 / 2}
$$

In Eq. (11), $n_{t o t}$ is the total cell-based number density and $\sigma_{v}$ is a reference collision cross section. In the simulations described below, we use $\sigma_{v}=5.81 \times 10^{-21} \mathrm{~m}^{2}$ along with constant values $Z_{\max }=91.5$ and $T^{*}=18.1 \mathrm{~K}$ in Eq. (7).

For a diatomic gas species $i$, the coefficient $C_{v}$ used to find the probability of vibrational-translational energy exchange is calculated as

$$
C_{v}=1+\frac{\frac{2}{3} \theta_{i} / T}{\exp \left(\theta_{i} / T\right)-1}
$$

after which the exchange probability is determined through the expression

$$
P_{v}=\frac{1}{C_{v}}\left[1-\exp \left(-C_{v} \frac{\Delta t}{\tau_{v}}\right)\right]
$$

If $P_{v}>R$, then vibrational-translational energy exchange will occur for this particle, and the vibrational energy $\varepsilon_{v}$ is resampled from a quantized equilibrium distribution at the particle temperature $T$

$$
\varepsilon_{\mathrm{v}}=\theta_{i} k_{B}\left\lfloor-\ln (R) T / \theta_{i}\right\rfloor
$$

where the operator \lfloor\rfloor rounds to the nearest smaller integer. Finally, once both rotational-translational and vibrational-translational energy exchange have been considered for this particle, the particle temperature is updated to enforce conservation of total energy.

$$
T \leftarrow T+\frac{\varepsilon_{r}^{o}+\varepsilon_{v}^{o}-\varepsilon_{r}-\varepsilon_{v}}{\frac{3}{2} k_{B}}
$$


In Eq. (15) the symbols $\varepsilon_{r}^{o}$ and $\varepsilon_{v}^{o}$ denote the initial rotational and vibrational energy values, respectively, before internal energy exchange operations.

Note that the final particle temperature calculated through Eq. (15) can potentially be negative. While a negative translational temperature is unphysical, all particle temperatures are reassigned to the cell temperature shortly afterward in step (4) of LD simulation procedures as outlined above, and the potentially negative particle temperature is used only in an evaluation of the cell-averaged translational temperature during step (1) of the LD procedures immediately after energy exchange operations. The resulting cell-averaged temperature is extremely unlikely to be negative, particularly if a reasonably large number of particles (at least 20) are assigned to the cell. However, to account for the unlikely occurrence of a negative cell temperature based on averaging among all particles in the cell, any cell-averaged temperature value less than zero is replaced by the temperature in this cell during the previous time step.

The energy exchange procedures involving Eqs. (6) through (15) provide a relatively simple means of including nonequilibrium rotational and vibrational energy distributions in continuum flow simulations using the LD particle method. Moreover, given the advantages of the LD particle method over other DSMC-based methods for continuum flow simulation, ${ }^{17-20}$ these energy exchange procedures constitute the first DSMC-based technique for inclusion of nonequilibrium internal energy distributions which also allows for accurate characterization of flow features such as slip lines or contact discontinuities when the time step and cell size are far too large for practical application of DSMC.

Another key benefit of the above energy exchange procedures is that - similar to the lack of dependence for LD method simulations on the mean collision time - rotation and vibrational equilibrium are automatically imposed when the time step interval $\Delta t$ is much larger than characteristic rotational and vibrational relaxation times. In contrast to a simple time integration of Eq. (1), the above procedures enforce a physically appropriate upper limit on the energy exchange probabilities (for rotational-translational energy exchange in a diatomic gas, this limit is 3/5) which tends to avoid instability or overshoot problems when a large ratio $\Delta t / \tau$ exists for either rotational or vibrational relaxation. Note however that the above assumptions of constant internal degrees of freedom and relaxation times may lead to errors when $\Delta t / \tau$ is large and considerable differences exist between translational and internal temperatures.

Despite these benefits of the energy exchange procedures involving Eqs. (6) through (15), one serious problem exists for these procedures when used as part of a hybrid LD-DSMC simulation: The mechanics of rotationaltranslational and vibrational-translational energy exchange as given above are significantly different than those used in DSMC. In particular, the Larsen-Borgnakke technique ${ }^{1}$ typically employed for energy exchange during inelastic DSMC collisions involves a different and more complex relationship between initial particle properties and postcollision internal energy distributions. Unlike the above procedures, the Larsen-Borgnakke technique creates a dependence for post-collision internal energy values on the initial internal energy of each particle participating in an inelastic collision. Moreover, inelastic collision probabilities in DSMC are commonly a function of the total relative energy in a binary collision, ${ }^{27}$ which produces a further dependence on the initial rotational or vibrational energy assigned to each particle. Additional capabilities developed for DSMC include consideration of collisional energy exchange within internal modes, as well as more physically based models for quantum transitions between individual vibrational energy levels. ${ }^{28-30}$ Such models rely on simulated binary collisions, and likely cannot be implemented through reasonably simple modifications to the energy exchange procedures described above.

As a result of these differences between DSMC and LD energy exchange, even if rotational and vibrational relaxation times are matched between DSMC and LD calculations in a hybrid LD-DSMC simulation utilizing the above energy exchange procedures, nonequilibrium characteristics of rotational and vibrational energy distributions may evolve discontinuously across the boundary between DSMC and LD domains. These nonequilibrium characteristics should not be noticeable in macroscopic output quantities such as the translational, rotational and vibrational temperatures, but any inconsistency in the treatment of nonequilibrium internal energy relaxation can potentially lead to some loss in simulation accuracy. This is particularly true if nonequilibrium features of the rotational and vibrational energy distributions are of interest, as in simulations used for gas radiation analysis.

\section{B. Second Approach}

To avoid discrepancies with DSMC in internal energy relaxation procedures as part of a hybrid LD-DSMC simulation, and to allow compatibility with improved relaxation models available for DSMC, an alternate approach for rotational and vibrational relaxation in the LD particle method has been developed. This second approach is simpler and easier to implement than the first approach described above, and uses the exact same energy exchange routines already employed in a preexisting DSMC code. However, the allowable time step size is limited by the 
rotational relaxation time for simulations involving rotational nonequilibrium, and as demonstrated below, this alternate approach for internal energy relaxation is considerably less efficient than the first approach.

In contrast to the first approach, here no additional calculations are performed before step (1) of the LD method procedures in the above outline. Instead, a series of further calculations are added between steps (3) and (4), just before new temperature and bulk velocity values are assigned to each particle. All cells are considered individually, and the following procedure is cycled through every cell within the LD domain. First, for all particles in a given cell, velocity components in the particle data structure used for particle movement are resampled from a Maxwellian distribution at the translational temperature $T_{c}$ assigned to the cell. All three velocity components $u_{k}(k=1,2,3)$ for each particle are computed as a function of two random numbers $R_{1}$ and $R_{2}$

$$
u_{k}=\left(-\ln \left(R_{1}\right) \frac{2 k_{B} T_{c}}{m_{i}}\right)^{1 / 2} \sin \left(2 \pi R_{2}\right)
$$

where $m_{i}$ is the molecular mass for the assigned particle species. Next, the average translational energy $e_{t}^{o}$ associated with particle velocities in the cell is calculated:

$$
e_{t}^{o}=\left\langle\frac{1}{2} m_{i} u_{k} u_{k}\right\rangle
$$

Standard DSMC procedures are then performed for assignment of collision partners among all particles in the cell. The only difference with respect to traditional collision selection in DSMC is that each collision probability (or equivalently, the number of potential collision pairs in Bird's no time counter scheme ${ }^{1}$ ) is multiplied by a factor $P_{\max }$ between zero and one, which is unique to this cell and is stored in the cell data structure. At simulation startup $P_{\max }$ is initialized for all cells at a small positive value much less than one (e.g. 0.01) and is subsequently increased, as discussed below, to match the maximum inelastic collision probability in each cell.

Once particles in this cell have been sorted as necessary into collision pairs, standard DSMC binary collision operations are performed. Velocity components $u_{k}$ and internal energy values $\varepsilon_{r}$ and $\varepsilon_{v}$ may be modified for each colliding particle. In an additional difference from traditional DSMC collisions, the probabilities $P_{r}$ and $P_{v}$ of rotational-translational and vibrational-translation energy exchange during each collision are divided by the factor $P_{\max }$ in the cell data structure. Noting that in most cases $P_{r} \gg P_{v}$ due to differences between rotational and vibrational collision numbers, we ensure that the conditions $P_{r} / P_{\max } \leq 1$ and $P_{v} / P_{\max } \leq 1$ are both generally satisfied by setting $P_{\max }=\min \left\{P_{r}, 1\right\}$ if $P_{r}>P_{\max }$ for any collision in this cell.

Next, an updated average translational energy $e_{t}$ in the cell is found by repeating the calculation in Eq. (17) with post-collision particle velocities $u_{k}$. The change in translational energy $e_{t}-e_{t}^{o}$ is then incorporated into the cell temperature

$$
T_{c} \leftarrow T_{c}+\frac{2}{3 k_{B}}\left(e_{t}-e_{t}^{o}\right)
$$

before this same temperature $T_{c}$ is assigned to all particles in the cell during step (4) of the LD method procedures. Note that velocity components $u_{k}$ used in particle movement are subsequently changed during step (5) of LD procedures, as outlined above.

In many continuum flows for which a nonequilibrium vibrational energy distribution may be of interest, the rotational energy distribution can reasonably be approximated as in a state of local equilibrium, with energy equipartition between rotational and translational degrees of freedom. This accounts for the widespread use of a single translational-rotational temperature in hypersonic CFD simulations, ${ }^{21}$ and allows rotational relaxation processes to be completely neglected with little expected impact on simulation accuracy. The same approximation may be employed here, to reduce the number of simulated binary collisions per time step and considerably improve simulation efficiency in any case where rotational-translational equilibrium can be assumed. Instead of using a variable probability $P_{r}$ of rotational-translational energy exchange based on the total collision energy, ${ }^{30}$ or using a temperature-based exchange probability equal to the reciprocal of the local rotational collision number, we instead set $P_{r}$ to a small constant value for all simulated collisions in every cell. This value must be larger than any vibrational-translational exchange probabilities $P_{v}$, and should also be sufficiency large to avoid significant differences between rotational and translational temperatures. Subject to these conditions, the constant $P_{r}$ value should be as small as possible for maximum efficiency; in the vibrational relaxation problem described below we

\section{8}

American Institute of Aeronautics and Astronautics Approved for public release; distribution unlimited. 
use $P_{r}=0.1$. Following the above procedures involving Eqs. (16) through (18), this means that all collision probabilities are multiplied by a factor 0.1 , all vibrational-translational exchange probabilities $P_{v}$ are divided by this same factor, and every collision results in rotational-translational energy exchange.

Note that use of a constant $P_{r}$ value does allow for finite rotational-translational relaxation rates, and may be used to approximate rotational nonequilibrium effects when such effects are considered less important than consistency with DSMC in vibrational relaxation processes. A constant $P_{r}$ value can also be used when a constant rotational-translational exchange probability is employed in DSMC calculations as part of a hybrid LD-DSMC simulation. In either case, $P_{r}$ should be set to the reciprocal of the rotational collision number.

\section{Model Evaluation}

To evaluate both approaches for rotational-translational and vibrational-translational energy exchange in the LD particle method, a series of homogeneous relaxation problems are simulated using each approach, and results are compared with those from DSMC and CFD simulations. For consistency with rotational relaxation times given by Eqs. (6) and (7), DSMC calculations here employ the variable exchange probability model of Boyd ${ }^{30}$ with the correction factor of Lumpkin et al. ${ }^{31}$ for rotational-translational energy exchange probabilities. For consistency with the vibrational relaxation time in Eq. (11), this equation is also used in DSMC calculations involving vibrational excitation to determine the relaxation time $\tau_{v}$ as a function of the instantaneous cell translational temperature. A uniform vibrational-translational exchange probability is calculated for all collisions in the cell during each time step, as the inverse product of the relaxation time $\tau_{v}$ and the collision frequency given by Eq. (6). The approximate correction factor of Gimelshein et al..$^{32}$ is then used to adjust the resulting exchange probability due to differences in the treatment of vibrational collision numbers between DSMC and continuum formulations. These same models for rotational-translational and vibrational-translational energy exchange are applied in LD simulations which employ the second approach to internal energy relaxation involving Eqs. (16) through (18). All CFD calculation results are taken from the published data of Deschenes et al., ${ }^{33}$ for which the same models and numerical parameters are used as in Eqs. (6), (7) and (11).

\section{A. Rotational Relaxation}

As a first test case to evaluate the proposed energy exchange procedures for the LD method, we consider a heat bath problem involving homogeneous rotational relaxation of $\mathrm{N}_{2}$ with no vibrational excitation. Two-dimensional simulations are performed on a $5 \times 5$ grid of square cells, bounded on all sides by symmetry boundaries. (While this is in principle a zero-dimensional problem, a two-dimensional treatment is employed here for reduced statistical scatter due to memory-based limitations on the number of particles per cell.) An average of about 2400 particles are assigned to each cell, for a total of around 60,000 particles. Following Deschenes et al., ${ }^{33}$ at simulation startup we impose a uniform translational temperature of $10,000 \mathrm{~K}$ and a rotational temperature of $1000 \mathrm{~K}$. Calculations are performed over a simulated time interval equal to 40 times the initial mean collision time $\tau_{\text {coll }}$.

Using the first LD method relaxation approach, which involves Eqs. (6) through (15) and is denoted for convenience as "LD1," we run simulations with a time step size corresponding to $\Delta t / \tau_{\text {coll }}=2$ and 10 . Using the second approach, denoted as "LD2," a single simulation is run with $\Delta t / \tau_{\text {coll }}=2$. (A time step ratio of 10 is not employed for the LD2 approach due to the very large corresponding number of simulated collisions per time step. To avoid an excessive number of collisions per time step in an LD2 simulation involving variable rotationaltranslational exchange probabilities $P_{r}$, the normalized time step $\Delta t / \tau_{\text {coll }}$ should be much smaller than the rotational collision number $Z_{r}$. In this case $Z_{r} \approx 13$ so $\Delta t / \tau_{\text {coll }} \ll 13$ for rotational relaxation using LD2.) For comparison, a DSMC simulation is performed with $\Delta t / \tau_{\text {coll }}=0.4$.

Simulations are run on a single $2.33 \mathrm{GHz}$ processor, and require the following calculation times: $0.74 \mathrm{~s}$ for LD1 with $\Delta t / \tau_{\text {coll }}=2 ; 0.20 \mathrm{~s}$ for LD1 with $\Delta t / \tau_{\text {coll }}=10 ; 2.30 \mathrm{~s}$ for LD2; and $3.94 \mathrm{~s}$ for DSMC. Thus, the two LD1 simulations are about $19 \%$ and $5 \%$ as expensive as the DSMC simulation, while the LD2 simulation is approximately 58\% as expensive as DSMC. Note in these comparisons that the primary efficiency advantage of the LD method over DSMC is associated with the use of much larger cells, and that simulation expense is independent of cell size in this type of homogeneous relaxation problem. Still, a comparison of simulation expense for this case demonstrates that, as expected, the LD1 approach is far more efficient than LD2, and LD2 is in turn more efficient than DSMC even when differences in cell size requirements are neglected.

In Fig. (1), translational and rotational temperatures, averaged over all 25 cells, are shown for all four simulations as a function of the normalized elapsed time $t / \tau_{\text {coll }}$. CFD results of Deschenes et al. ${ }^{33}$ are also included in Fig. (1) for comparison. As expected, we find very good agreement with both DSMC and CFD temperature profiles 
for all three LD simulations, with a maximum difference from published CFD data of around $170 \mathrm{~K}$ in rotational temperature for the LD2 case.

Figure (2) shows the rotational energy distribution from LD and DSMC simulations at the elapsed time $t / \tau_{\text {coll }}=$ 10. Particle rotational energy values $\varepsilon_{r}$ are sorted into bins of width $\Delta \varepsilon_{r}=6.05 \times 10^{-20} \mathrm{~J}$, and the rotational energy distribution for each simulation is approximated as a histogram with both axes nondimensionalized by the bin width $\Delta \varepsilon_{r}$. The Boltzmann rotational energy distribution $f_{B}\left(\varepsilon_{r}\right)$ for a diatomic gas, corresponding to equilibrium conditions at the rotational temperature $T_{r}$ from the DSMC simulation, is also shown for comparison. ${ }^{1}$

$$
f_{B}\left(\varepsilon_{r}\right)=\frac{1}{k_{B} T_{r}} \exp \left(-\frac{\varepsilon_{r}}{k_{B} T_{r}}\right)
$$

Rotational energy distributions for all four simulations are shown in Fig. (2) to diverge considerably from the Boltzmann distribution, with particularly large disagreement at higher energy levels. In contrast, the LD2 distribution is found to agree very well with DSMC, subject to significant statistical scatter at high energy levels, whereas relatively poor agreement is observed between DSMC and LD1 results. As discussed above, differences in nonequilibrium rotational relaxation between LD1 and DSMC are expected, and likely occur as a result of differences in the underlying mechanics of rotational-translational energy exchange.

Similar results are found in Fig. (3), which shows corresponding rotational energy distributions at the normalized elapsed time $t / \tau_{\text {coll }}=20$. In all simulations, the energy distribution is found here to better approximate the equilibrium distribution than in Fig. (2), due to the increased time for the relaxation process at $t / \tau_{\text {coll }}=20$. Still, significant disagreement is found in Fig. (3) between DSMC and both LD1 simulations, particularly at the higher energy levels. As in Fig. (2), excellent agreement is observed in Fig. (3) between the DSMC and LD2 distributions. Figure (4) shows rotational energy distributions at the final elapsed time $t / \tau_{\text {coll }}=40$. As shown in Fig. (1), rotational and translational temperatures are nearly equal at this elapsed time, and the rotational relaxation process has progressed for sufficiently long that the rotational energy distribution from all four simulations is found to closely approximate the equilibrium Boltzmann distribution. Small discrepancies are still observed at high rotational energy levels in Fig. (4) between LD1 results and DSMC, particularly for the LD1 simulation with $\Delta t / \tau_{\text {coll }}=10$, although the size of these discrepancies is comparable to the level of statistical scatter.

In evaluating the results from Figs. (1) through (4), we conclude that both LD1 and LD2 approaches provide good agreement with DSMC for the variation in rotational and translational temperatures during homogeneous rotational relaxation, and both approaches allow for nonequilibrium rotational energy distributions at intermediate times while converging toward the appropriate Boltzmann distribution. However, significant differences are found between LD1 and DSMC results in the rotational energy distributions at intermediate times. The increased efficiency of the LD1 approach relative to LD2 is therefore balanced by a corresponding reduction in consistency with DSMC.

\section{B. Vibrational Relaxation}

To evaluate vibrational-translational energy exchange in the LD1 and LD2 procedures, we use a similar test case of Deschenes et al. ${ }^{33}$ involving homogeneous vibrational relaxation. This case is identical to the previous case, except here the gas is vibrationally excited, and at simulation startup both rotational and translational temperatures are initialized at $10,000 \mathrm{~K}$ while the vibrational temperature is initialized at $1000 \mathrm{~K}$. LD method simulations are performed using both LD1 and LD2 approaches with a normalized time step size of $\Delta t / \tau_{\text {coll }}=10$. Following an assumption of near-equilibrium between rotational and translational energy modes, we impose a constant rotationaltranslational exchange probability $P_{r}=0.1$ in the LD2 simulation, as described above, to avoid an excessively large number of simulated collisions per time step. A DSMC simulation is performed with $\Delta t / \tau_{\text {coll }}=0.4$ for comparison, and all simulations are run to a final elapsed time of $t / \tau_{\text {coll }}=4000$. Simulation run times are $27.4 \mathrm{~s}$ for LD1, $59.1 \mathrm{~s}$ for LD2, and $479 \mathrm{~s}$ for DSMC. LD1 and LD2 simulations are therefore about $6 \%$ and $12 \%$ as expensive, respectively, as the DSMC simulation.

In Fig. (5), translational and vibrational temperatures are plotted as a function of normalized time $t / \tau_{\text {coll }}$ for all three simulations, and temperature profiles are compared with published CFD results of Deschenes et al. ${ }^{33}$ Excellent agreement is observed in Fig. (5) between all results, with maximum relative differences under $1 \%$ for both translational and vibrational temperature. Although not shown in the figure, rotational temperatures from all simulations are practically indistinguishable from translational temperatures, so the assumption in the LD2 simulation of rotational-translational equilibrium is found to be valid as expected. 
Figure (6) shows the quantized vibrational distribution function from LD1, LD2 and DSMC simulations at the normalized elapsed time $t / \tau_{\text {coll }}=400$. Also shown for comparison is the equilibrium Boltzmann distribution $f_{B}\left(i_{v}\right)$ at the DSMC vibrational temperature $T_{v}$

$$
f_{B}\left(i_{v}\right)=\left[1-\exp \left(-\theta / T_{v}\right)\right] \exp \left(-i_{v} \theta / T_{v}\right)
$$

where $i_{v}$ is the quantum vibrational level and $\theta$ is the characteristic temperature for vibrational excitation. ${ }^{34}$ All simulation data are found here to be in poor agreement with the Boltzmann distribution, signifying a large departure from vibrational equilibrium. Reasonably good agreement is observed between all three simulation results, although the agreement with DSMC is significantly better for the LD2 simulation than for LD1. In particular, we find a noticeable underestimate, relative to DSMC, in LD1 vibrational energy level populations at $i_{v}=1,2$ and 3 , and a corresponding overestimate at $i_{v}=0$ and $i_{v} \geq 6$. In contrast, discrepancies observed between LD2 and DSMC data points are very small, and are likely dominated by statistical scatter.

Similar trends are found in Figs. (7) and (8), which show vibrational energy distributions at elapsed times $t / \tau_{\text {coll }}=$ 800 and 4000 respectively. In comparing Fig. (7) with Fig. (6), we find that, as expected, the simulated vibrational energy distributions more closely approximate an equilibrium distribution when a longer period is allowed for vibrational relaxation. As in Fig. (6) all three simulation results in Fig. (7) differ considerably from the Boltzmann distribution, particularly at the higher energy levels. While the agreement in Fig. (7) between LD1 and DSMC results is somewhat better than that shown in Fig. (6), there is still noticeably better agreement in Fig. (7) between DSMC and LD2 than between DSMC and LD1.In Fig. (8), which displays the vibrational energy distributions at the final time $t / \tau_{\text {coll }}=4000$, sufficient time has elapsed in the relaxation process for the vibrational energy distribution to converge to the equilibrium solution. Small differences from the Boltzmann limit, particularly at higher energy levels where the sampling size is very small, can be attributed mainly to statistical scatter.

Figure (9) shows variation with time in relative populations for the bottom six vibrational energy levels. Excellent agreement is found here between DSMC and LD2 results, even though the time step size is 25 times larger in the LD2 simulation than in DSMC. In contrast, only fair agreement is found between DSMC and LD1; the largest discrepancy is around $t / \tau_{\text {coll }}=100$ where the relative population at energy level $i_{v}=1$ is roughly $35 \%$ lower in the LD1 simulation. At intermediate periods during the relaxation process, the LD1 simulation is observed to underestimate vibrational energy level populations relative to DSMC at $i_{v}=1,2$, and 3 , while overestimating populations at $i_{v}=0$ and $i_{v} \geq 5$.

In comparing calculated vibrational energy distributions with DSMC, it should be emphasized that the LarsenBorgnakke model used for vibrational-translational energy exchange in the DSMC calculations is inherently phenomenological. This model is intended to allow relaxation toward equilibrium while satisfying detailed balance, without any basis in the underlying physics. While the LD1 approach has no more physical basis than the LarsenBorgnakke model, a better gauge of accuracy for LD1 results would be a comparison with available experimental data in a similar relaxation problem. Still, consistency with DSMC is desired for inclusion in a DSMC-based hybrid algorithm, and the comparison with DSMC shown in Figs. (6) through (9) is useful both for assessing such consistency in the LD1 approach and for verifying that the LD2 approach can successfully reproduce DSMC results even when the time step size is well out of the practical range for DSMC. Moreover, LD2 should be compatible with any technique for vibrational-translational energy exchange developed for DSMC, so the very good agreement found between LD2 and DSMC results in Figs. (6) through (9) should extend to more sophisticated DSMC models which include additional phenomena or use fewer approximations to the underlying physics. ${ }^{28,29}$

\section{Hypersonic Flow Demonstration}

To demonstrate capabilities of the LD-DSMC hybrid code with modifications for internal energy nonequilibrium, additional simulations are performed for a test case involving hypersonic flow over a cylinder. This case is based on that in Ref. 18, with $\mathrm{N}_{2}$ flow at a global Knudsen number of 0.01 , but here the gas is vibrationally excited and the freestream Mach number has been increased from 6 to 15. As in Ref. 18, the cylinder surface is modeled as a specularly reflecting wall, and viscous modifications to the LD particle method described in Ref. 19 are not used. Thus, no boundary layer is assumed to form along the cylinder surface, and viscous transport effects are neglected in LD calculations within continuum regions. This lack of consideration for viscous effects is necessitated by the fact that particles in the LD domain travel along streamlines at the gas bulk velocity, so that even when viscous flow simulation procedures outlined in Ref. 19 are employed, no diffusive transport occurs for rotational and vibrational energy. This means that unphysical transverse gradients in rotational and vibrational 
temperatures may be found within boundary layer regions in the LD domain, particularly where diffusive transport effects dominate effects of advective transport and translational-internal energy exchange. While the viscous LD method modifications described in Ref. 19 should still be usable with rotational and vibrational nonequilibrium routines in cases (such as free expansion flows) where internal energy nonequilibrium is expected only outside of boundary layers, inviscid flow must be assumed for the present case to avoid problems associated with a lack of consideration for diffuse transport in internal energy modes.

Hybrid LD-DSMC simulations are performed using both LD1 and LD2 approaches, with a constant $P_{r}$ value of 0.2 in the LD2 simulation, and a DSMC simulation is also run for comparison. Both hybrid simulations use a standard gradient length local Knudsen number $\mathrm{Kn}_{\mathrm{GLL}}$ for assessment of continuum breakdown. ${ }^{3}$ This number is defined as the ratio of the local mean free path to the minimum value among characteristic length scales based on translational temperature, density or bulk velocity. The parameter $\mathrm{Kn}_{\mathrm{GLL}}$ is periodically (every 2000 time steps) evaluated in each grid cell, and a cutoff value of 0.05 is used for assignment of cells to either LD or DSMC domains. Freestream inflow conditions include a temperature of $217.5 \mathrm{~K}$ and number density of $1.6 \times 10^{21} \mathrm{~m}^{-3}$.

All simulations use the same grid with 45,000 cells refined to less than twice the local mean free path, and DSMC subcells with dimensions of one-half the mean free path are employed to further reduce the mean separation distance between colliding DSMC particles. The time step interval is automatically and dynamically adapted in each cell, so that the local Courant number is no larger than 0.2 and a maximum of around $20 \%$ of the particles within a cell collide during each time step. Numerical weight values are also automatically adapted to allow for at least 60 particles per cell.

For each simulation, sampling is performed over 30,000 time steps, following an additional 30,000 time steps during a startup period required for convergence to steady state. Simulations are run on eight processors in the nyx cluster at the University of Michigan. Hybrid simulations require a total of 100.5 and 103.2 CPU hours, for calculations involving LD1 and LD2 internal nonequilibrium approaches respectively, while the DSMC simulation requires 48.3 CPU hours. Although a comparison with DSMC of simulation expense may seem very discouraging, it should be emphasized that the primary motivation for the hybrid LD-DSMC scheme is to allow for much larger cells and time step sizes in continuum regions than is possible using DSMC. As this case is used only for a preliminary demonstration of code capabilities in a multidimensional flow, these advantages of the LD-DSMC scheme are not utilized, and both cell dimensions and time step intervals within the LD domain are sufficiently small to meet DSMC guidelines.

Contours of pressure from all three simulations are shown in Fig. 10. Continuum breakdown boundaries which separate LD and DSMC domains, as taken from the hybrid LD1 simulation, are also shown in the figure. As expected, continuum breakdown occurs in a narrow region surrounding the bow shock, and through much of the wake region downstream of the cylinder. Some scatter is observed in the continuum breakdown boundary, particularly in the far wake region at $x>0.2 \mathrm{~m}$, but this is thought to have no significant effect on simulation accuracy. In comparing pressure contour lines from the three simulations, we find excellent agreement throughout the simulated flowfield. Some small differences are observed, particularly along the outflow boundary on the right side, in the wake region near the symmetry boundary at $\mathrm{y}=0 \mathrm{~m}$, and at the downstream edge of the shock around $\mathrm{y}$ $=0.1 \mathrm{~m}$. Maximum local pressure differences are roughly equal to the $5 \%$ error tolerance corresponding to the breakdown parameter cutoff value of 0.05 .

In Fig. 11, contours of Mach number from the three simulations are shown along with streamlines and LDDSMC domain boundaries from the LD1 simulation. Differences in contour line locations are more noticeable in Mach number than in pressure, although the most visible differences here occur in regions of relatively small Mach number gradients. In particular, variation in the $\mathrm{M}=3$ contour lines between $\mathrm{x}=0.1$ and $0.2 \mathrm{~m}$ correspond to local differences in Mach number of less than 3\%. In observing this same set of contour lines for $\mathrm{M}=3$, we find a small but noticeable discontinuity in slope along the LD-DSMC domain boundary for both the LD1 and LD2 simulations. This can be explained by the fact that the boundary lies almost directly along a streamline in a region of significant transverse velocity gradients. Thus, the inherent differences in diffusive momentum transport between the LD and DSMC methods should result in slope discontinuities along this boundary. When viscous flow simulation procedures in the LD method are enabled, the increased diffusive transport in LD calculations is found to prevent such discontinuities. However, as discussed above, these procedures must be disabled here to avoid problems associated with a lack of diffusive transport for internal energy modes.

Translational temperature contours from all three simulations are shown in Fig. 12. As with Mach number contours in Fig. 11, Fig. 12 shows good overall agreement and some clear differences in contour line locations. These differences are most noticeable along the forebody surface, in the wake, and in the post-shock region far from the axis. In all of these regions, however, translational temperature gradients are relatively small, and maximum local differences in translational temperature are only around $4 \%$.

12

American Institute of Aeronautics and Astronautics Approved for public release; distribution unlimited. 
In Fig. 13, gas bulk velocity and pressure are plotted along the stagnation streamline as functions of distance from the cylinder surface. Excellent agreement is found for both quantities for all three simulations, with extremely small differences (less than 1\%) in shock standoff distance and post-shock stagnation pressure. The largest differences in pressure are found at the upstream end of the shock, where the DSMC result shows a more gradual slope than either hybrid simulation result. This can be partly explained by the fact that the edge of the shock is located along a boundary between DSMC and LD domains, and inviscid flow is assumed in the LD calculations. In an inviscid flow the shock is ideally represented by a pressure discontinuity, not a gradual pressure increase as in DSMC, so the inviscid flow assumption should have some influence on gas properties within any LD cells that border the shock. The magnitude of the disagreement here could likely be reduced by decreasing the cutoff value for the continuum breakdown parameter $\mathrm{Kn}_{\mathrm{GLL}}$, in order to extend the DSMC domain further upstream around the shock. A reduction in the cutoff value also warranted by the fact that a $\mathrm{Kn}_{\mathrm{GLL}}$ value of 0.05 (as is used here) has previously been used in hybrid CFD-DSMC hypersonic flow simulations which employ a Navier-Stokes solver in continuum regions, ${ }^{3}$ while the present continuum calculations neglect viscous effects and are strictly valid only at the low Knudsen number limit.

Figure 14 shows the variation in translational, rotational and vibrational temperatures along the stagnation streamline. While very good agreement between the three simulations is observed within the DSMC regions surrounding the shock, significant differences are found in the subsonic shock layer region further downstream. For both in the hybrid LD-DSMC simulations, translational and rotational temperatures are underpredicted in this postshock region by around 3\%. Note that, as expected from the excellent agreement in pressure shown in Fig. 13, gas density is overpredicted in this region by approximately $3 \%$. The level of disagreement increases toward the wall, where translational and rotational temperatures are underpredicted by between $5 \%$ and $8 \%$ in the hybrid simulations. The increased discrepancies along the wall can be primarily attributed to a lack of diffusive transport for vibrational energy in the LD calculations, which allows for large gradients in vibrational temperature within the stagnant flow region near the wall. In contrast, these diffusive effects are likely the dominant transport mechanism around the stagnation point in the DSMC simulation, as indicated by the plateau in DSMC vibrational temperature within the same near-wall region where the greatest disagreement is observed between DSMC and hybrid temperature profiles.

Further upstream, in the post-shock region within the LD domain in the hybrid simulations, a significant underprediction in vibrational temperature is found in the hybrid simulation results in Fig. 14. One possible cause for this underprediction is the assumption of translational equilibrium in cells within the LD domain just downstream of the shock. The determination of continuum breakdown using gradient-based parameters such as $\mathrm{Kn}_{\mathrm{GLL}}$ is inherently approximate, and an alternate breakdown parameter or smaller cutoff value may be necessary to reduce vibrational temperature discrepancies in this post-shock region.

Figure 15 shows the variation along the stagnation streamline in the fraction of particles assigned to the first excited vibrational energy level $\left(i_{v}=1\right)$. As with vibrational temperature, both LD1 and LD2 curves in Fig. 15 show large overpredictions relative to DSMC in the stagnant flow region around the wall. The DSMC curve levels off in this region as a result of diffusive transport effects, while the lack of diffusive transport for vibrational energy in the LD calculations allows the post-shock gradient in this quantity to continue up to the cylinder surface. Further upstream within the post-shock region, relatively good agreement is observed between LD2 and DSMC simulation results. In contrast, a significant underprediction is found here in the LD1 result. These trends are consistent with those shown in Figs. 6 through 9 for the homogeneous vibrational relaxation problem; considerable differences are found between LD1 and DSMC in relaxation rates for individual vibrational energy levels, while LD2 and DSMC results for these relaxation rates agree very well. Thus, as in LD2 simulations of the homogeneous relaxation problems, the reduced efficiency relative to LD1 is balanced by an improvement in accuracy for modeling vibrational nonequilibrium effects.

\section{Conclusions}

Two different approaches have been presented for inclusion of nonequilibrium internal energy distributions in the LD particle method. Both approaches allow continuous rotational and discrete vibrational distributions, with probabilistic rotational-translational and vibrational-translational exchange procedures and finite relaxation rates. In the first approach, denoted as LD1, relaxation occurs over an arbitrarily large time step interval through a single resampling procedure for some fraction of particles in each computational cell. This provides a very efficient means of energy exchange, and permits arbitrary internal energy distributions under nonequilibrium conditions while automatically satisfying energy equipartition at equilibrium for any time step size. In the second approach, termed LD2, simulated binary collisions are used to redistribute energy between translational and internal modes, following energy exchange procedures in DSMC. While collision-based procedures make LD2 less efficient than LD1, a 
modification to the calculated collision probabilities results in less stringent limitations on time step size than in standard DSMC. Time step limitations in LD2 are relaxed even further for LD2 when rotational equilibrium can be maintained using a small constant rotational-translational exchange probability, or when a constant exchange probability can be assumed in modeling rotational relaxation effects.

To evaluate the two LD energy exchange approaches, a series of LD and DSMC simulations were performed for homogeneous rotational and vibrational relaxation problems. Temperature profiles and internal energy distributions were compared between LD1, LD2, DSMC, and available CFD data. As expected, very good agreement with DSMC and CFD was found in rotational, vibrational and translational temperature profiles for both LD1 and LD2. Both approaches also were found to allow large departures from equilibrium in the rotational and vibrational energy distributions, although agreement with DSMC in these nonequilibrium distributions was significantly better for LD2 than LD1.

In a demonstration of current code capabilities, both LD1 and LD2 approaches were applied in hybrid LDDSMC simulations of a Mach 15 flow of $\mathrm{N}_{2}$ over a cylinder. Reasonably good agreement with results from a DSMC simulation was observed for most flow quantities considered, although some significant discrepancies were found. Many of these discrepancies can be attributed to inviscid flow assumptions in LD calculations, particularly the lack of diffusive transport models for internal energy modes in the LD method. Due in part to the lack of current capabilities in the LD method for diffusive internal energy transport, these simulations involved a specularly reflecting cylinder surface and did not consider boundary layer effects.

When used as part of a hybrid scheme with DSMC, both LD1 and LD2 approaches offer significant advantages over rotational and vibrational nonequilibrium capabilities in a hybrid CFD-DSMC algorithm. ${ }^{33}$ In particular, there is no loss of information related to internal energy distributions along the boundary between LD and DSMC domains. In addition, the LD2 approach allows any internal energy exchange procedures developed for DSMC to be implemented for use in continuum flow calculations. This last advantage seems potentially the most promising, as high enthalpy continuum flows may involve significant internal nonequilibrium - particularly for vibrational energy - but existing CFD models for inclusion of nonequilibrium internal energy distributions ${ }^{22}$ are far more limited than those developed for DSMC. ${ }^{27-29}$ While it should generally be less efficient than LD1, the LD2 approach is simpler to implement, ensures consistency with DSMC internal energy exchange procedures in a hybrid simulation, and permits extension of DSMC energy exchange models to continuum flow problems where DSMC is prohibitively expensive and other DSMC-based continuum methods give excessive numerical diffusion. Furthermore, LD2 offers the potential, as planned in future work, of incorporating DSMC chemistry models for application to continuum flows.

Other proposed areas of future work include detailed assessment of both accuracy and efficiency for the hybrid scheme, through comparison with DSMC for a series of hypersonic blunt body and multiscale expansion flows with viscous effects. Work currently in progress includes the implementation of diffusive transport models for rotational and vibrational energy, with diffusive fluxes based on gradients of time-averaged translational, rotational and vibrational temperatures. Other current work involves modifications to DSMC procedures so that each grid cell may be split into a large number of dynamically adaptive subcells, and so that cell-based time step and numerical weight values are automatically set to allow a relatively uniform number of particles per subcell. This should permit small mean separation distances between colliding DSMC particles even when each cell is far larger than the local mean free path. With uniformly much larger cells in both DSMC and LD regions, a significant efficiency increase should be found in hybrid LD-DSMC hypersonic flow simulations through the use of fewer particles per cell and larger time step intervals within the LD domain.

\section{Acknowledgments}

Financial support for this work was provided in part by Spectral Sciences, Inc. through contract W9113M-06-C0122, a Missile Defense Agency (MDA) Small Business Innovative Research (SBIR) Phase II award. The authors gratefully acknowledge Jason Cline and Matt Braunstein for their oversight of this work. Additional financial support was provided by NASA through grant NNX08AD02A.

\section{References}

${ }^{1}$ Bird, G. A., Molecular Gas Dynamics and the Direct Simulation of Gas Flows, Clarendon Press, Oxford, 1994.

${ }^{2}$ Schwartzentruber, T. E., and Boyd, I. D., "A Hybrid Particle-Continuum Method Applied to Shock Waves," Journal of Computational Physics, Vol. 215, 2006, pp. 402-416.

${ }^{3}$ Schwartzentruber, T. E., and Boyd, I. D., "A Modular Particle-Continuum Numerical Method for Hypersonic Nonequilibrium Gas Flows,” Journal of Computational Physics, Vol. 225, 2007, pp. 1159-1174. 
${ }^{4}$ Lian, Y.-Y., Wu, J.-S., Cheng, G., and Koomullil, R., "Development of a Parallel Hybrid Method for the DSMC and NS Solver," AIAA Paper 2005-0435, 2005.

${ }^{5}$ Kaplan, C. R., Liu, J., and Oran, E. S., "Parallel Hybrid Method for Subsonic Flows: Coupling and Load-Balancing Challenges," AIAA Paper 2006-992, 2006.

${ }^{6}$ Garcia, A. L., Bell, J. B., Crutchfield, W. Y., and Alder, B. J., "Adaptive Mesh and Algorithm Refinement Using Direct Simulation Monte Carlo," Journal of Computational Physics, Vol. 154, 1999, pp. 134-155.

${ }^{7}$ Wadsworth, D. C., and Erwin, D. A., "Two-Dimensional Hybrid Continuum/Particle Approach for Rarefied Flows," AIAA Paper 92-2975, 1992.

${ }^{8}$ Hash, D. B., and Hassan, H. A., "Assessment of Schemes for Coupling Monte Carlo and Navier-Stokes Solution Methods," Journal of Thermophysics and Heat Transfer, Vol. 10, No. 2, 1996, pp. 242-249.

${ }^{9}$ Roveda, R., Goldstein, D. B., and Varghese, P. L., "Hybrid Euler/Direct Simulation Monte Carlo Calculation of Unsteady Slit Flow," Journal of Spacecraft and Rockets, Vol. 37, No. 6, 2000, pp. 753-760.

${ }^{10}$ Breuer, K. S., Piekos, E. S., and Gonzales, D. A., "DSMC Simulations of Continuum Flows,” AIAA Paper 95-2088, 1995.

${ }^{11}$ Titov, E. V., Zeifman, M. I., and Levin, D. A., "Application of the Kinetic and Continuum Techniques to the Multi-Scale Flows in MEMS Devices," AIAA Paper 2005-1399, 2005.

${ }^{12}$ Pullin, D. I., "Direct Simulation Methods for Compressible Inviscid Ideal-Gas Flow," Journal of Computational Physics, Vol. 34, 1980, pp. 231-244.

${ }^{13}$ Macrossan, M. N., "A Particle-Only Hybrid Method for Near-Continuum Flows," Rarefied Gas Dynamics: $22^{\text {nd }}$ International Symposium, American Institute of Physics, 2001, pp. 388-395.

${ }^{14}$ Tiwari, S., and Klar, A., "An Adaptive Domain Decomposition Procedure for Boltzmann and Euler Equations," Journal of Computational and Applied Mathematics, Vol. 90, 1998, pp. 223-237.

${ }^{15}$ Burt, J. M., and Boyd, I. D., "Evaluation of a Particle Method for the Ellipsoidal Statistical Bhatnagar-Gross-Krook Equation," AIAA Paper 2006-989, 2006.

${ }^{16}$ Macrossan, M. N., "The Equilibrium Flux Method for the Calculation of Flows with Non-equilibrium Chemical Reactions," Journal of Computational Physics, Vol. 80, 1989, pp. 204-231.

${ }^{17}$ Burt, J. M., and Boyd, I. D., “A Low Diffusion Particle Method for Simulating Compressible Inviscid Flows," Journal of Computational Physics, Vol. 227, 2008, pp. 4653-4670.

${ }^{18}$ Burt, J. M., and Boyd, I. D., "A Hybrid Particle Approach for Continuum and Rarefied Flow Simulation," Journal of Computational Physics, Vol. 228, 2009, pp. 460-475.

${ }^{19}$ Burt, J. M., and Boyd, I. D., "Extension of a Multiscale Particle Scheme to Near-Equilibrium Viscous Flows," AIAA Journal, 2009, to appear (accepted for publication).

${ }^{20}$ Burt, J. M., and Boyd, I. D., “Application of a Multiscale Particle Scheme to High Altitude Rocket Exhaust Flows,” AIAA Paper 2009-1567, 2009.

${ }^{21}$ Scalabrin, L. C., and Boyd, I. D., "Development of an Unstructured Navier-Stokes Solver For Hypersonic Nonequilibrium Aerothermodynamics," AIAA Paper 2005-5203, 2005.

${ }^{22}$ Landrum, D. B., and Candler, G. V., "Development of a New Model for Vibration-Dissociation Coupling in Nitrogen," AIAA Paper 92-2853, 1992.

${ }^{23}$ Parker, J. G., "Rotational and Vibrational Relaxation in Diatomic Gases," Physics of Fluids, Vol. 2, 1959, pp. 449-462.

${ }^{24}$ Jeans, J. H., The Dynamical Theory of Gases, Cambridge University Press, London, 1916.

${ }^{25}$ Millikan, R. C., and White, D. R., "Systematics of Vibrational Relaxation," Journal of Chemical Physics, Vol. 39, No. 12, 1963, pp. 3209-3213.

${ }^{26}$ Park, C., "Problems of Rate Chemistry in the Flight Regimes of Aeroassisted Orbital Transfer Vehicles," AIAA Paper 841730, 1984.

${ }^{27}$ Boyd, I. D., “Analysis of Rotational Nonequilibrium in Standing Shock Waves of Nitrogen,” AIAA Journal, Vol. 28 , No. 11, 1990, pp. 1997-1999.

${ }^{28}$ Bondar, Y. A., and Ivanov, M. S., "Resonant VV Exchange in the DSMC Method with the Larsen-Borgnakke Model," AIAA Paper 2008-3918, 2008.

${ }^{29}$ Vijayakumar, P., Sun, Q., and Boyd, I. D.," Vibrational-translational Energy Exchange Models for the Direct Simulation Monte Carlo Method," Physics of Fluids, Vol. 11, No. 8, 1999, pp. 2117-2126.

${ }^{30}$ Boyd, I. D., "Relaxation of Discrete Rotational Energy Distributions Using a Monte Carlo Method," Physics of Fluids A, Vol. 5, No. 9, 1993, pp. 2278-2286.

${ }^{31}$ Lumpkin, F. E., Haas, B. L., and Boyd, I. D., "Resolution of Differences Between Collision Number Definitions in Particle and Continuum Simulations," Physics of Fluids A, Vol. 3, No. 9, 1991, pp. 2282-2284.

${ }^{32}$ Gimelshein, N. E., Gimelshein, S. F., and Levin, D. A., "Vibrational Relaxation Rates in the Direct Simulation Monte Carlo Method," Physics of Fluids, Vol. 14, No. 12, 2002, pp. 4452-4455.

${ }^{33}$ Deschenes, T. R., Holman, T. D., Boyd, I. D., and Schwartzentruber, T. E., “Analysis of Internal Energy Transfer Within a Modular Particle-Continuum Method," AIAA Paper 2009-1213, 2009.

${ }^{34}$ Vincenti, W. G., and Kruger, C. H., Introduction to Physical Gas Dynamics, Krieger Publishing Company, Malabar, Florida, 1986. 


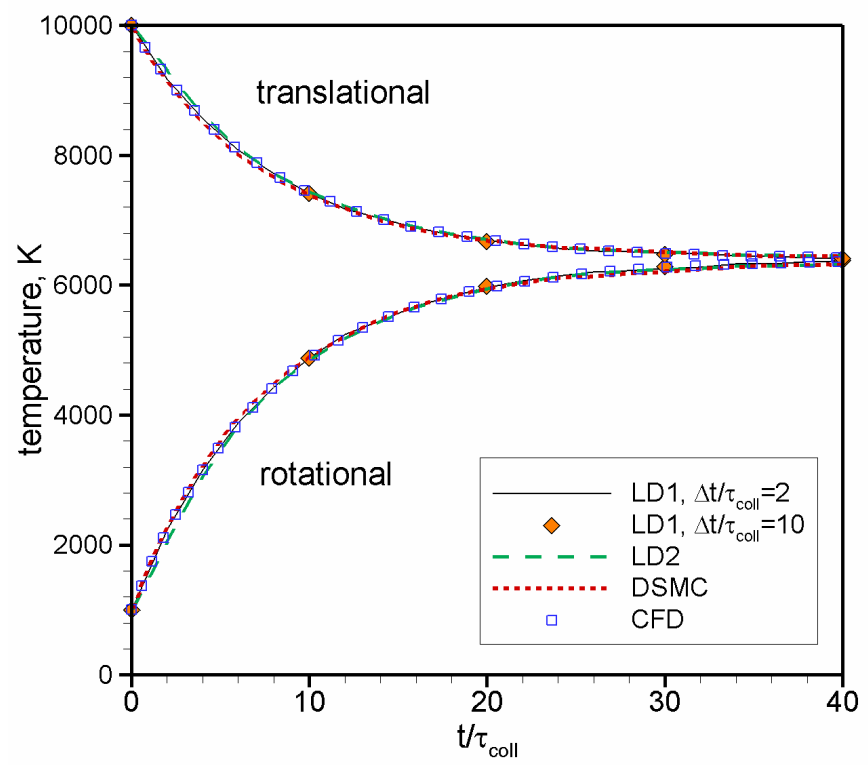

Figure 1. Variation in rotational and translational temperatures for homogeneous rotational relaxation in $\mathbf{N}_{2}$.

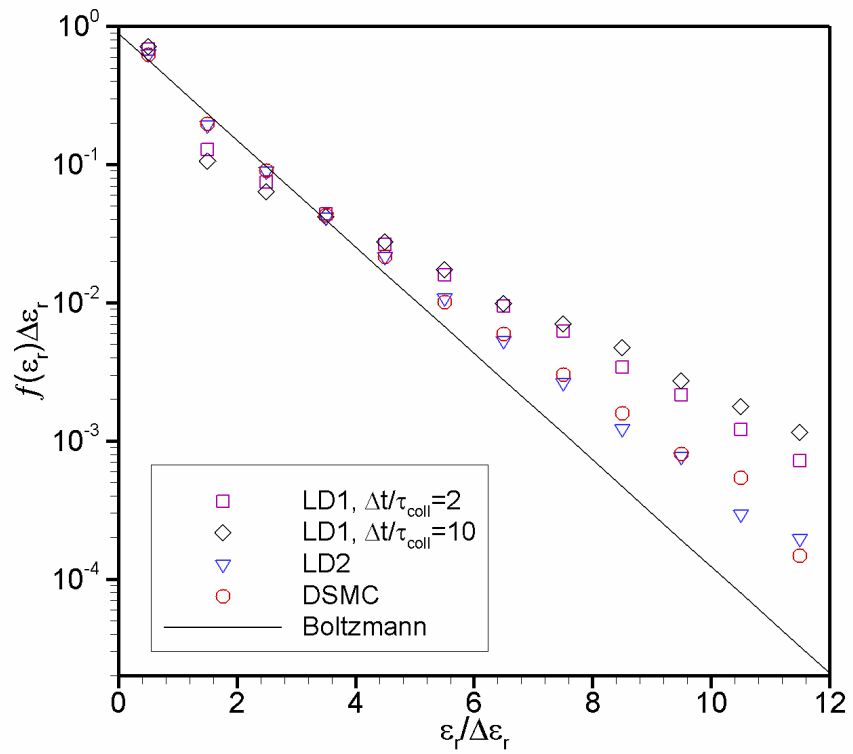

Figure 2. Rotational energy distribution function at the elapsed time $t / \tau_{\text {coll }}=10$. 


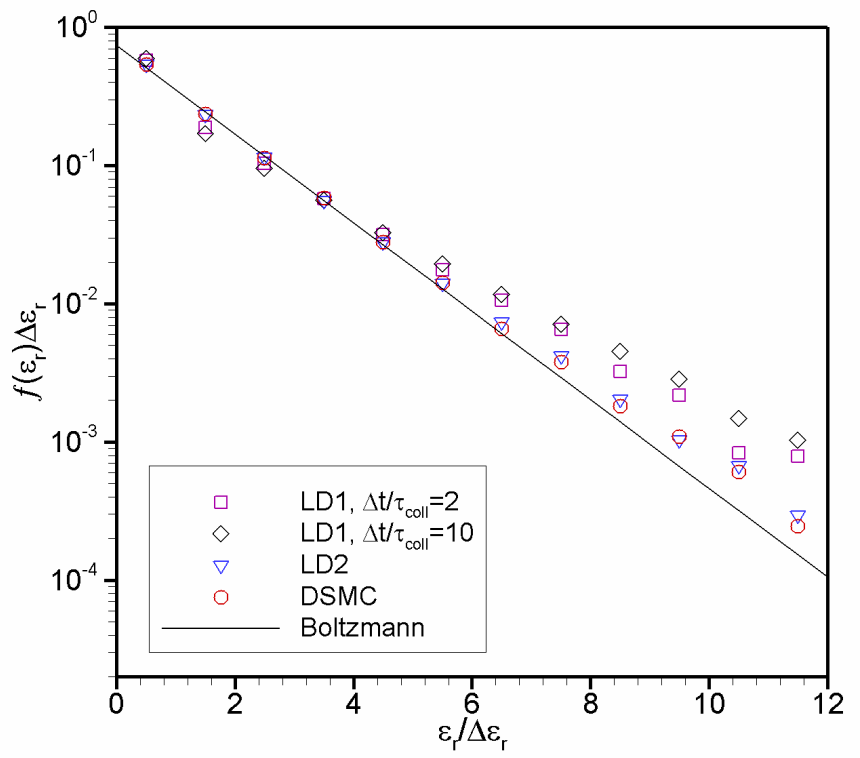

Figure 3. Rotational energy distribution function at $t / \tau_{\text {coll }}=\mathbf{2 0}$.

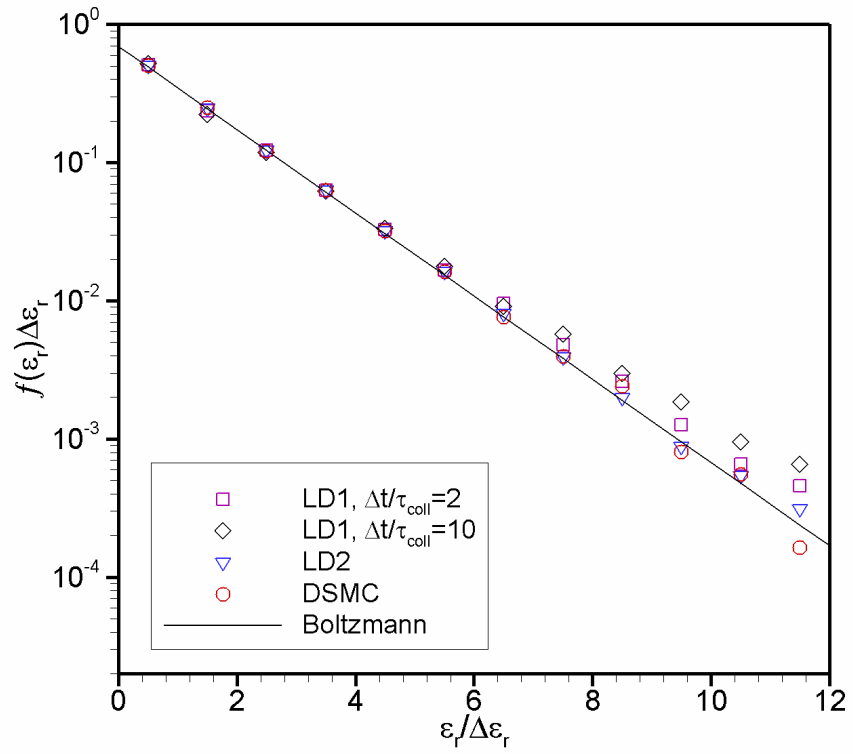

Figure 4. Rotational energy distribution function at $t / \tau_{\text {coll }}=40$. Approved for public release; distribution unlimited. 


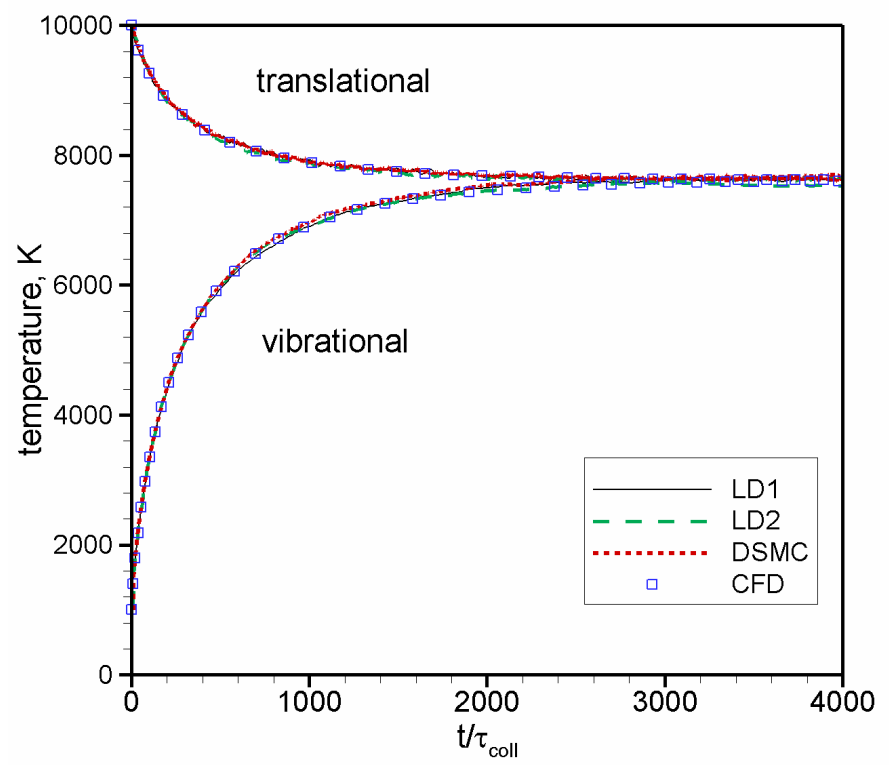

Figure 5. Variation in vibrational and translational temperatures for homogeneous vibrational relaxation in $\mathrm{N}_{2}$.

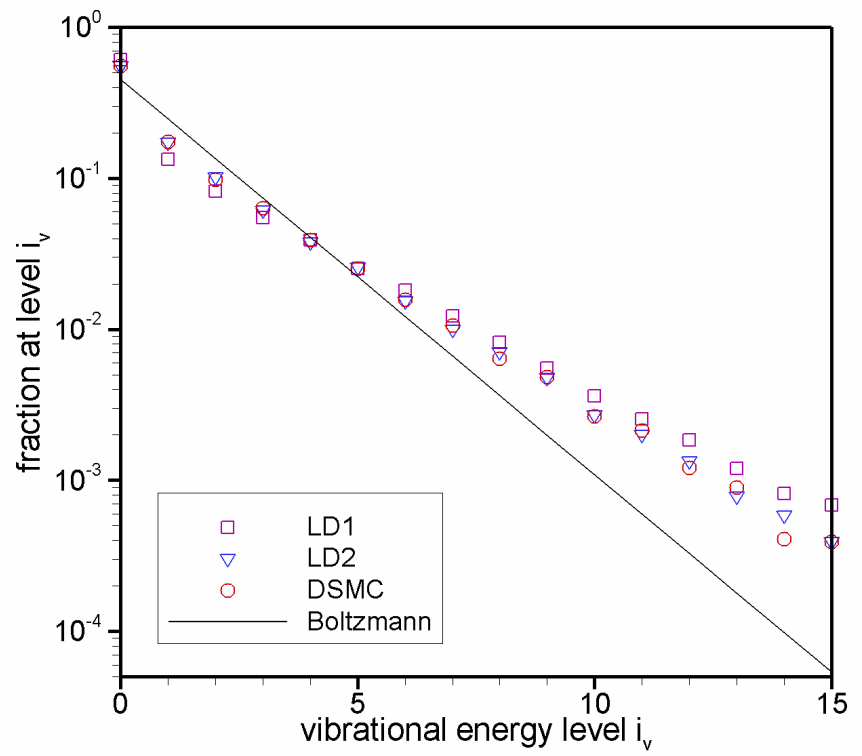

Figure 6. Vibrational energy distribution function at $t / \tau_{\text {coll }}=400$. 


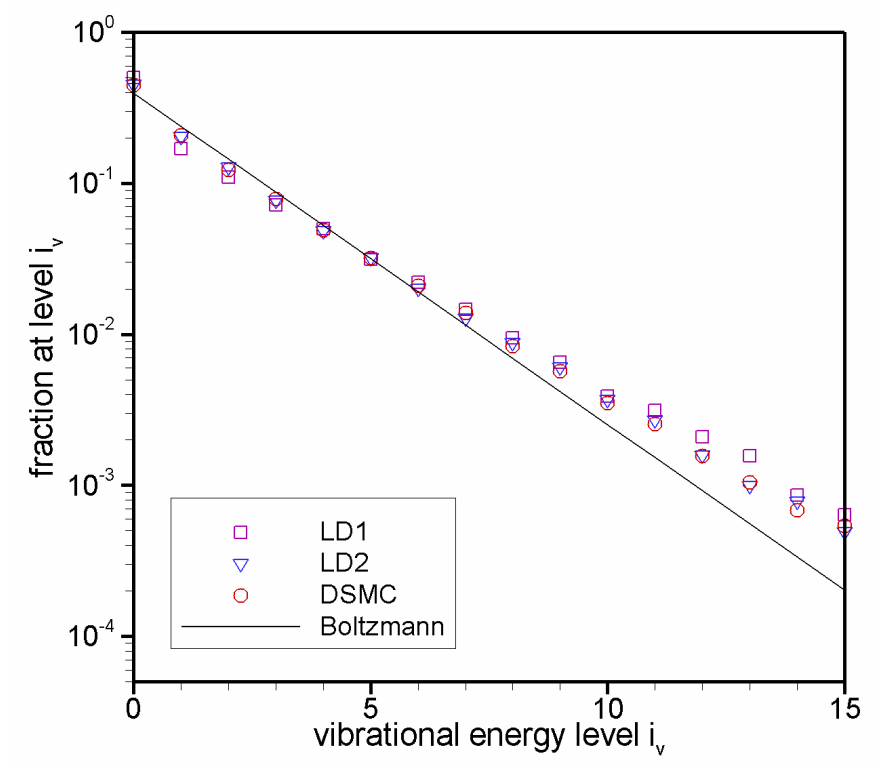

Figure 7. Vibrational energy distribution function at $t / \tau_{\text {coll }}=800$.

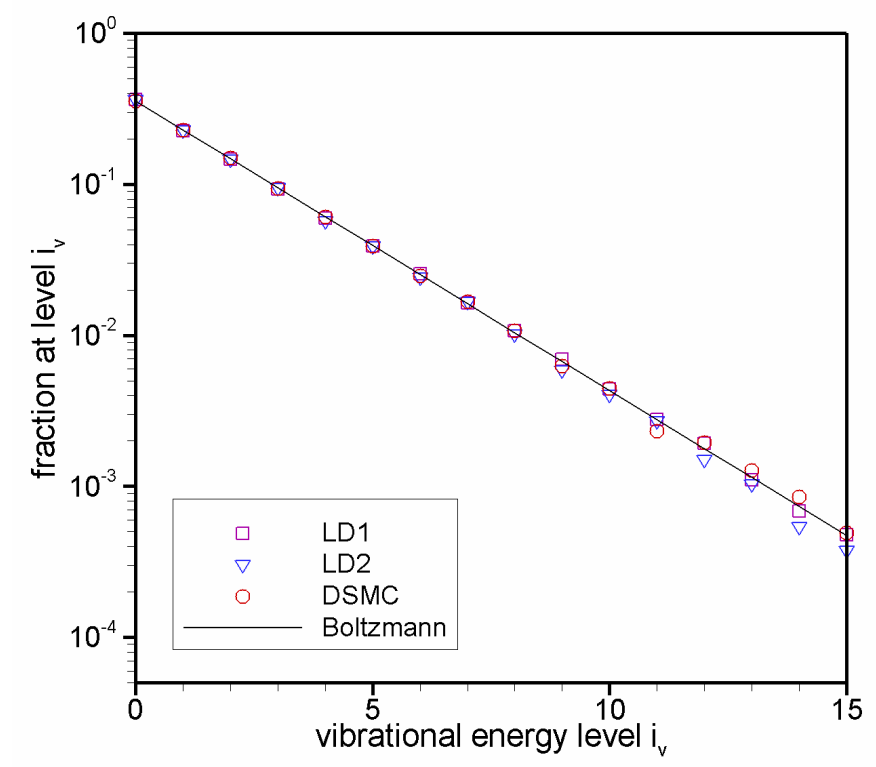

Figure 8. Vibrational energy distribution function at $t / \tau_{\text {coll }}=4000$. 


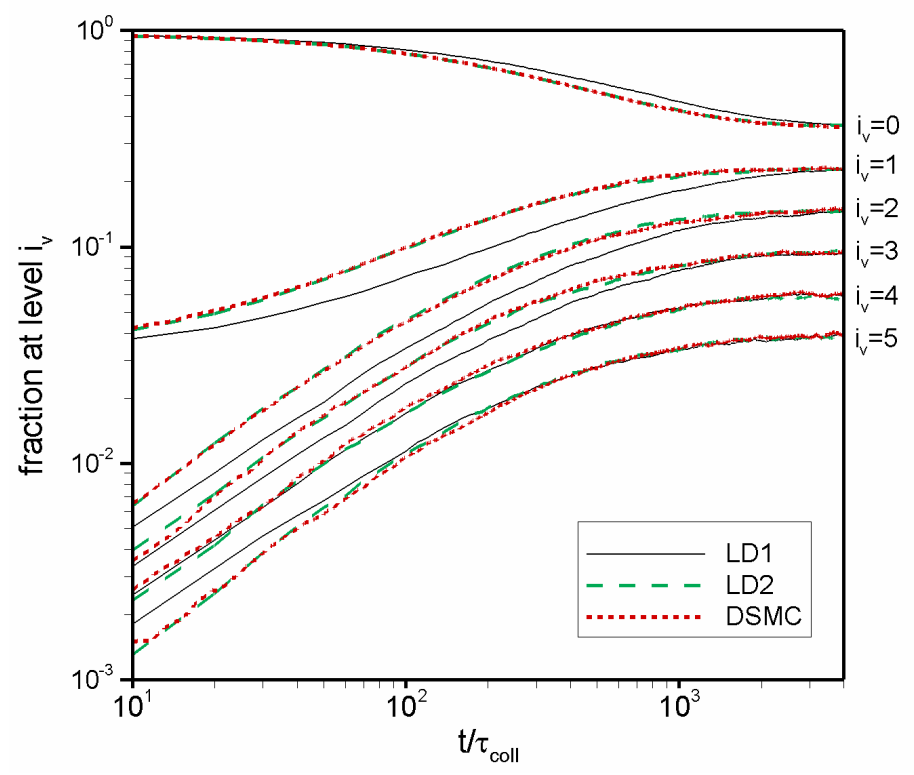

Figure 9. Time variation in relative populations for vibrational energy levels 0 through 5.

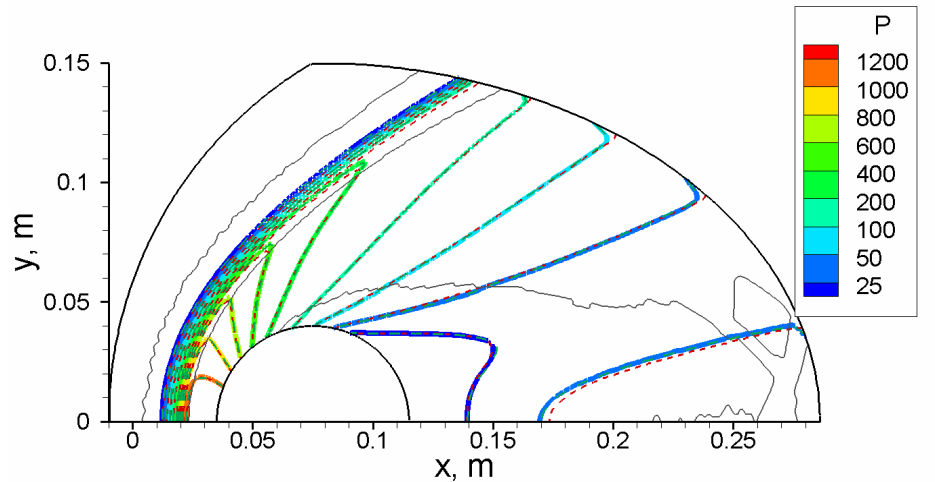

Figure 10. Contours of pressure, in units of Pa, for a Mach 15 flow of $\mathrm{N}_{2}$ over a cylinder. Multicolored lines are from the LD1 hybrid simulation, green dashed lines are from the LD2 simulation, and red dotted lines are from DSMC. Thin black lines denote LDDSMC domain boundaries in the LD1 simulation. 


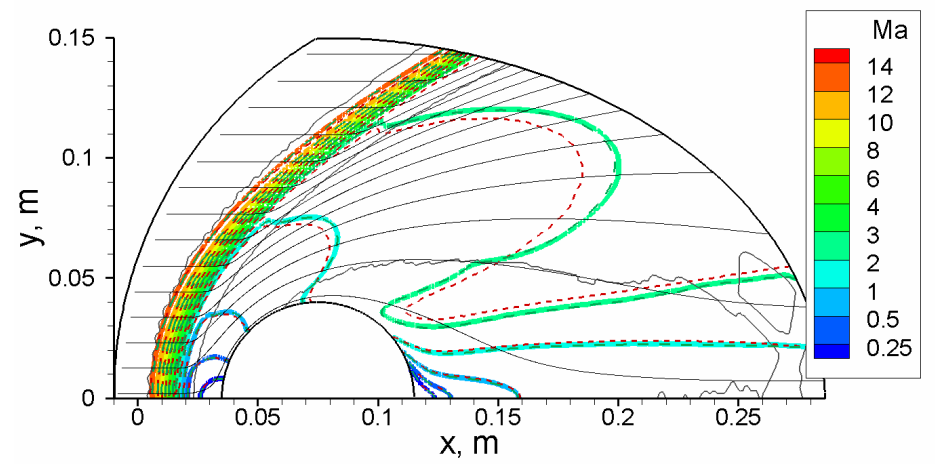

Figure 11. Contours of Mach number from LD1, LD2 and DSMC simulations, and streamlines from the LD1 simulation. Line conventions are as in Fig. 10.

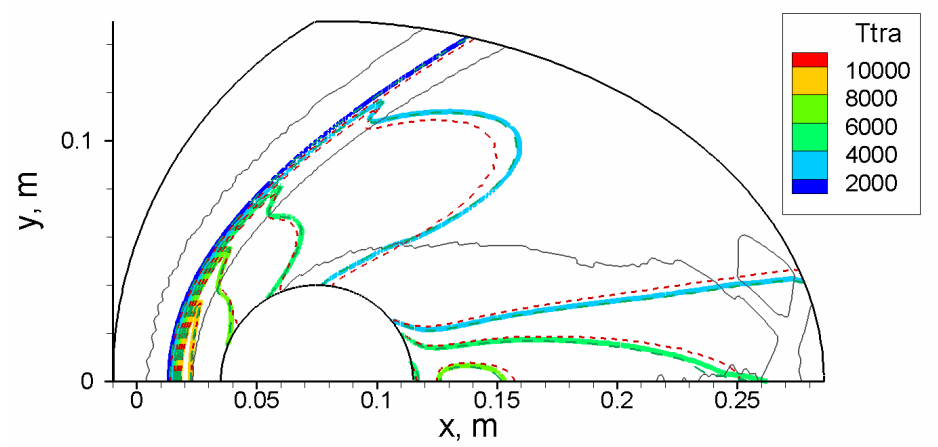

Figure 12. Contours of translational temperature from LD1, LD2 and DSMC simulations. Legend values are in units of $K$. 


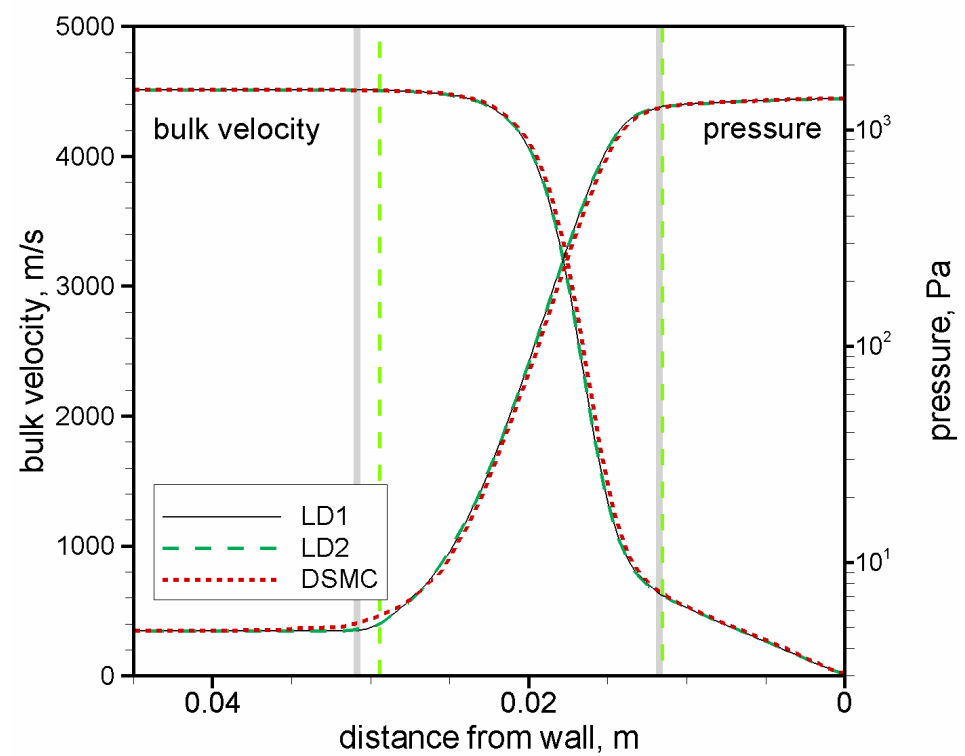

Figure 13. Variation in bulk velocity and pressure along the stagnation streamline. Solid and dashed vertical lines indicate LDDSMC domain boundaries from LD1 and LD2 simulations respectively.

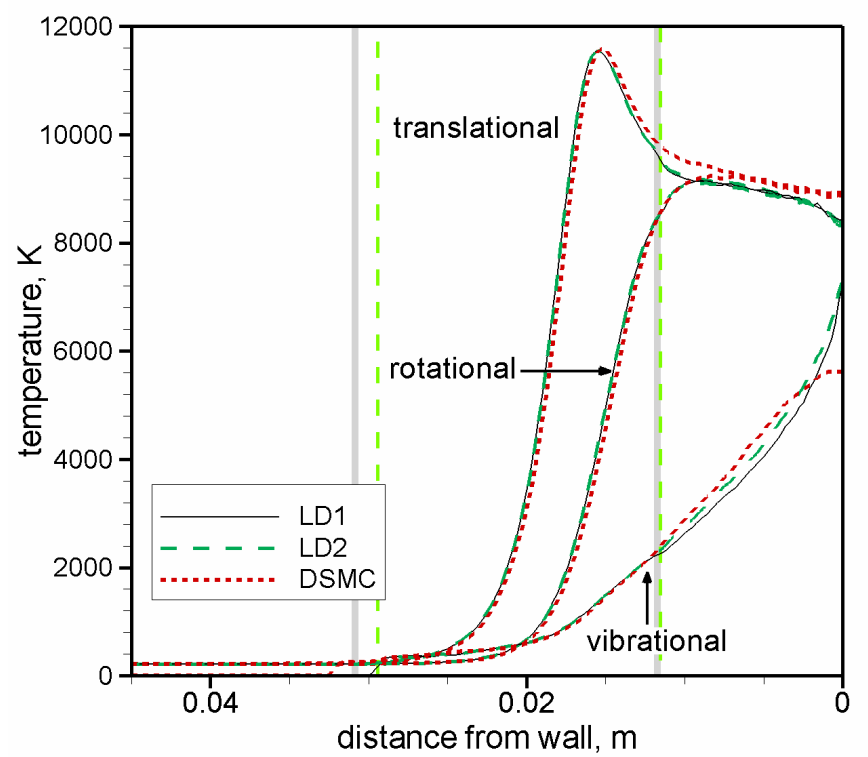

Figure 14. Translational, rotational and vibrational temperatures along the stagnation streamline. 


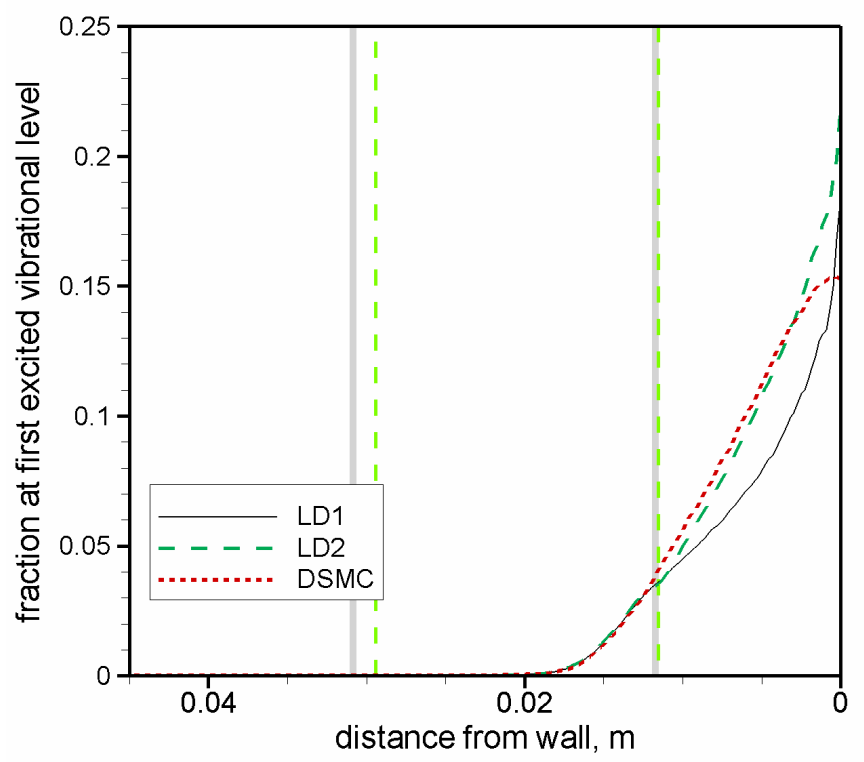

Figure 15. Variation along the stagnation streamline in the fraction of particles at the first excited vibrational level.

American Institute of Aeronautics and Astronautics Approved for public release; distribution unlimited. 